\title{
Blunted Stress Response in Small for Gestational Age Neonates
}

\author{
LEONHARD SCHÄFFER, DEBORAH MÜLLER-VIZENTINI, TILO BURKHARDT, MANFRED RAUH, ULRIKE EHLERT, \\ AND ERNST BEINDER
}

\author{
Department of Obstetrics [L.S., D.M.-V., T.B., E.B.], Institute of Psychology [U.E.], University of Zürich 8091, Switzerland; Department of \\ Pediatrics [M.R.], University of Erlangen 91052, Germany
}

\begin{abstract}
There is evidence that adverse conditions during intrauterine development affect future health of the offspring. Hypothalamus-pituitary-adrenal (HPA) axis dysregulation is assumed to play an important role in the association of small for gestational age (SGA) and the pathogenesis of hypertension and the metabolic syndrome. Stress response patterns in SGA neonates may identify a link with intrauterine-induced permanent maladaptation of the HPA axis. Salivary cortisol and cortisone levels were therefore analyzed during resting conditions and in response to a pain-induced stress event in SGA ( $<5$ th percentile) and appropriate for gestational age (AGA) neonates born $\geq 34$ wk of gestation. In AGA neonates, salivary cortisol and cortisone levels significantly increased after the stress event $(p<0.05)$. In contrast, SGA infants exhibited a blunted steroid release after stress induction $(p=0.76, p=0.65$, respectively). No influence of mode of delivery $(p=0.93)$, gender $(p=$ $0.21)$, and gestational age $(p=0.57)$ on stress response patterns was observed in a multiple stepwise regression. SGA neonates show a blunted physiologic activation of the HPA axis in response to a stress stimulus. Thus, intrauterine-induced alteration of HPA axis regulation seems to persist into the postnatal period and represents a prerequisite for the hypothesis of HPA axis involvement in the fetal origin of adult diseases. (Pediatr Res 65: 231-235, 2009)
\end{abstract}

$\mathrm{S}$ ubstantial evidence from epidemiologic data and experimental animal models indicate that stressful conditions during intrauterine fetal life affect behavioral development and future health of the offspring. Maladaptation of the fetal autonomic regulation has been suggested as a putative predisposing factor in the pathogenesis of cardiovascular, metabolic, and psychiatric diseases in low birth weight (LBW) infants (1-3). This has been reinforced by the discovery of an early programming of the stress response-mediating neuroendocrine system, namely the hypothalamic-pituitary-adrenal (HPA) axis and the autonomic nervous system $(4,5)$. As such, LBW, a surrogate for fetal malnutrition has been linked with increased adrenocortical hormone activity and activation of the sympathetic nervous system (6-9) in childhood and adult life in independent populations.

A pathophysiologic explanation of this phenomenon described by Barker's group as "fetal programming" (10) has been derived from animal models revealing long-term changes in the molecular expression of steroid receptors within the limbic system (11).

Received June 11, 2008; accepted September 18, 2008

Correspondence: Leonhard Schäffer, M.D., Universitäts-Frauenklinik, Frauenklinikstrasse 10, CH-8091 Zürich, Switzerland; e-mail: leonhard.schaeffer@usz.ch
Increases in glucocorticoids, such as cortisol and cortisone, are generally considered as adaptive mechanisms to mobilize energy for survival in response to a stressful event or to danger (12). Prolonged intrauterine exposure to stress, however, may lead to an alteration of the set point of the stress regulatory system resulting in increased levels of cortisol that can negatively effect the functioning of several biologic systems $(13,14)$ and ultimately result in arterial hypertension and diabetes mellitus type II, one of the main risk factors for arteriosclerosis-associated diseases.

Because patterns of stress response in early postnatal life may help to identify prenatally induced alterations in the HPA axis and simultaneously exclude an only temporarily activated HPA axis due to adverse intrauterine conditions, we analyzed the stress reactivity of the HPA axis in small for gestational age (SGA) newborns. Our results provide evidence that the HPA axis is already altered in SGA newborns and may, if these alterations persist permanently, play a role in the intrauterine origin of adult diseases.

\section{MATERIALS AND METHODS}

Subjects. The study was approved by the Research Ethics Committee of the University of Zurich and the Federal Ethics Commission of the Canton of Zurich. Written maternal consent was obtained from all participants in the study.

Saliva samples from 40 appropriate for gestational age (AGA) and 18 SGA infants were analyzed. Approximately, 40 SGA infants after 34 wk of gestation were born during the study period (April-September, 2005) in our hospital with 2300 deliveries per year. Healthy controls were recruited until approximately the double number of study patients was reached to obtain adequate numbers for statistical evaluation. SGA was defined as birth weight below the 5th percentile of the gender-specific newborn reference chart (15). Birth weight between the 10th and 90th percentile was required for AGA children. Only healthy newborns delivered after 34 wk of gestation ( $\geq 238 \mathrm{~d})$ were included in the study. Newborns requiring intensive care or those with malformations were excluded from the study. Additional exclusion criteria included maternal substance abuse (nicotine, alcohol) and exogenous corticosteroid treatment (e.g., for lung maturation induction) during pregnancy. Sonographic measurements of fetal crown-rump length and biparietal diameter during the first trimester served as parameters for accurate determination of gestational age. In the SGA group, 10 infants had a diagnosis of intrauterine growth restriction (IUGR) established either by serial sonographic biometry or by pathologic Doppler measurements, one was SGA alone and 7 infants were referred to our clinic for delivery only and did not receive antenatal care at our institution. Sonographic data for the latter group were not available.

Experimental procedures. Salivary cortisol and cortisone levels were collected between 72 and $96 \mathrm{~h}$ postnatal during a routine heel prick for the Guthrie test (a newborn screening test for metabolic disorders). This was considered to be the equivalent of a stress event. Although infants are born

Abbreviations: AGA, appropriate for gestational age; HPA axis, hypothalamus-pituitary-adrenal axis; SGA, small for gestational age 
without a circardian rhythm and acquire it only within the first year of life (16), saliva samples were collected only during the morning hours between 8 a.m. and 1 p.m. from each infant $10 \mathrm{~min}$ before and $20 \mathrm{~min}$ after the stress event. Baseline levels were obtained in an undisturbed environment. The time of collection was based on experiments revealing peak cortisol responses between 20 and 30 min postmanipulation (17). To obtain the saliva, a cotton swab was placed in the infants' mouth for a duration of 5 min. Salivary cortisol reflects the unbound, active fraction of cortisol and is highly correlated with plasma cortisol levels $(18,19)$. Samples were placed in saliva collection tubes (Salivette, Sarstedt, Nümbrecht, Germany) and frozen at $-20^{\circ} \mathrm{C}$ until further analysis.

Cortisol/cortisone measurements. Saliva cortisol and cortisone were determined simultaneously using liquid chromatography tandem mass spectrometry, with atmospheric pressure chemical ionization in the positive ion mode, according to a modified method of Rauh et al. (20). One hundred microliters of the samples and calibrators were deproteinized with methanol/ zinc sulfate $(50 \mathrm{~g} / \mathrm{L}, 1 / 1 \mathrm{vol} / \mathrm{vol})$. After centrifugation, the supernatants were applied to an online solid-phase extraction column with subsequent highperformance liquid chromatography separation using column switching (extraction column: Oasis HLB $2.1 \times 20 \mathrm{~mm}, 15 \mu \mathrm{m}$; Waters, Milford, MA). The samples were washed with 5\% methanol and eluted in back-flush with 2 $\mathrm{mM}$ ammonium acetate/methanol (30:70, vol/vol) onto the analytical column (Chromolith RP $18 \mathrm{e} 100 \times 4.6 \mathrm{~mm}$, Merck, Darmstadt, Germany) at a flow rate of $1 \mathrm{~mL} / \mathrm{min}$. Sample analysis was performed in the multiple-reaction monitoring mode with a dwell time of $150 \mathrm{~ms}$ per channel using the following transitions for quantification (qualifier transition): $\mathrm{m} / \mathrm{z}$ 363.2/121.2 (363.2/ 309.4) cortisol, $\mathrm{m} / \mathrm{z}$ 361.1/162.9 (361.1/239.0) cortisone, $\mathrm{m} / \mathrm{z}$ 367.3/121.2 Cortisol-d4.

Statistical analysis. We applied STATA 9 Statistics/Data Analysis Software (Stata Corporation, College Station, TX) for statistical analysis according to Altman's recommendations for repeated measurements (21). Baseline characteristics of SGA and AGA infants were compared using the MannWhitney test and $\chi^{2}$ test when appropriate. Because basal levels of cortisol vary considerably in individual newborns and children (17), absolute values and relative alterations were analyzed. Cortisol and cortisone values were not normally distributed as analyzed by the Shapiro-Francia W' test. We therefore analyzed the difference between cortisol and cortisone baseline levels and time point "20 min post" using the Wilcoxon signed rank test. The MannWhitney test was used for comparison of raw baseline cortisol and cortisone levels. A stepwise multiple regression was applied to test for putative influencing factors on cortisol alterations such as gestational age, birth weight independent of gestational age, mode of delivery (vaginal/cesarean section), and gender. To validate our statistical analyses, a power calculation was conducted in SGA infants assuming the mean increase in cortisol levels of AGA infants as relevant. Finally, cortisol and cortisone levels in AGA and SGA infants were tested for correlation using the Spearman rank correlation test. The level of statistical significance of all analyses was set at $p<0.05$.

\section{RESULTS}

The gestational ages of the AGA and SGA newborns were comparable (median: 273 versus $266 \mathrm{~d}$ of gestation, respectively, $p=0.29$ ). Median birth weight of the SGA children was $2265 \mathrm{~g}$ corresponding to the 1.6th weight percentile compared with $3288 \mathrm{~g}$ corresponding to the $52 \mathrm{nd}$ percentile in the AGA group. A summary of the characteristics of the study population is given in Table 1.

Median levels for basal cortisol and cortisone were comparable in the AGA and SGA neonates (1.18 versus $0.95 \mathrm{ng} / \mathrm{mL}$, $p=0.7 ; 11.35$ versus $13.55 \mathrm{ng} / \mathrm{mL}, p=0.42$, respectively). Individual stress responses are illustrated in Figure $1 a$ and $b$. In AGA neonates, salivary levels of cortisol and cortisone significantly increased after the stress stimulus (2.4 and 18.15 $\mathrm{ng} / \mathrm{mL}, p<0.05$, respectively). In contrast, the cortisol and cortisone response in SGA neonates was noticeably blunted $(0.69 \mathrm{ng} / \mathrm{mL}, p=0.76$ and $13.05 \mathrm{ng} / \mathrm{mL}, p=0.65$, respectively) (Fig. $2 a$ and $b$ ). To validate the absent cortisol response in SGA neonates a one-sample t test power calculation was conducted for the given sample size $(n=18)$ revealing a power of $99 \%$ at a significance level of 0.05 .
Table 1. Baseline characteristics

\begin{tabular}{lccc}
\hline & AGA & SGA & $p$ \\
\hline Gestational age (d) & $273(240-294)$ & $266(240-292)$ & 0.289 \\
Birth weight (g) & $3288(2100-3780)$ & $2265(1470-2870)$ & $<0.05$ \\
Weight percentile & $52(17.6-91.6)$ & $1.6(0.1-4.3)$ & $<0.05$ \\
Body length (cm) & $49.5(45-54)$ & $45(40-48)$ & $<0.05$ \\
Length percentile & $65.2(5.3-100)$ & $3.85(0-35.2)$ & $<0.05$ \\
Gender, & $18 / 22$ & $6 / 12$ & 0.697 \\
$\quad$ male/female & & & \\
Delivery, & & & \\
$\quad$ vaginal/cesarean & & & \\
5-min APGAR & $9(8-10)$ & $9(8-10)$ & 0.307 \\
$\quad$ score & & & \\
Maternal age (yr) & $30(18-39)$ & $29(19-39)$ & 0.839 \\
Parity & $2(1-4)$ & $1(1-4)$ & $<0.05$ \\
\hline
\end{tabular}

Values are medians with ranges in parentheses; $n=40$ appropriate for gestational age (AGA) neonates and 18 small for gestational age (SGA) neonates.
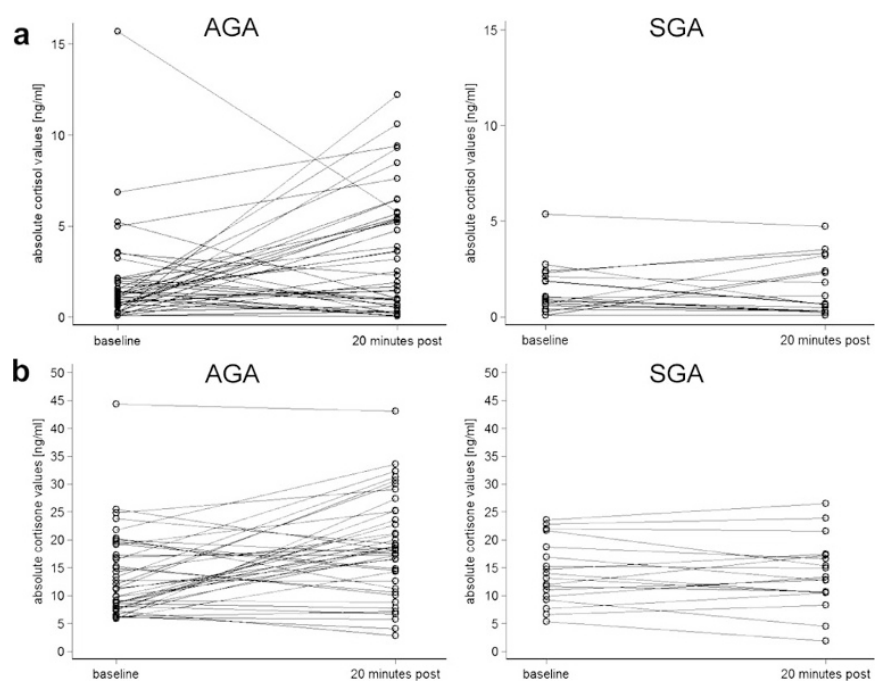

Figure 1. Salivary absolute levels for cortisol $(a)$ and cortisone $(b)$ including individual courses of AGA $(n=40)$ and SGA $(n=18)$ newborns before (baseline) and after (20 min post) application of the stress stimulus.
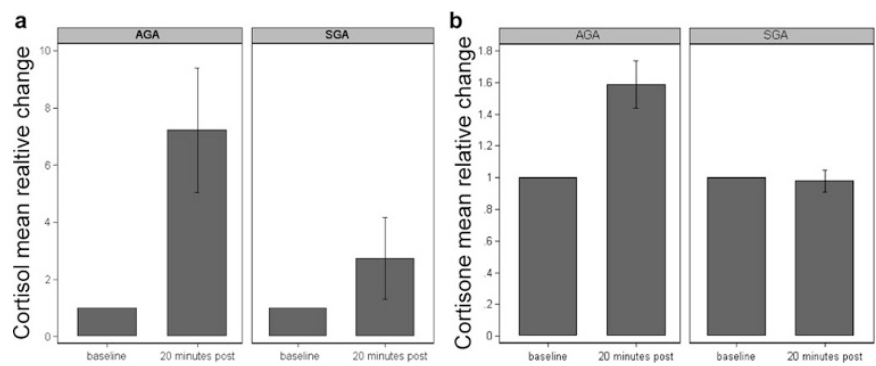

Figure 2. Mean relative salivary level alterations for cortisol $(a)$ and cortisone $(b)$ and SEM. In AGA newborns $(n=40)$, cortisol and cortisone levels significantly increase after stress induction $(p<0.05)$. In SGA newborns $(n=$ 18), cortisol and cortisone levels do not significantly increase after application of the stress stimulus ( $p=0.76, p=0.65$, respectively).

To test for a putative influence of gestational age, gender, birth weight, and mode of delivery on cortisol stress response patterns a multiple stepwise regression model was applied. None of these factors were found to have a significant influence $(p=0.574, p=0.207, p=0.347, p=0.927$, respectively, Table 2). 
Table 2. Multiple stepwise regression model

\begin{tabular}{lcc}
\hline & Regression coefficient & Standard error \\
\hline Gestational age & -0.0246 & 0.0458 \\
Birth weight & 0.0011 & 0.0011 \\
Gender & 0.8982 & 0.9786 \\
Mode of delivery & 0.0947 & 1.0211 \\
\hline
\end{tabular}

Stepwise regression of putative confounding factors on HPA axis activity. The stepwise backward regression started with this full model, the criterion for removing a variable was $p \geq 0.200$. Adjusted $R^{2}$ for full model: -0.0220 .

Finally, a Spearman rank correlation revealed a strong correlation for cortisol and cortisone levels in AGA $(R=0.77$, $p<0.001)$ and in SGA $(R=0.82, p<0.001)$ neonates, thus excluding the possibility that decreased cortisol responses in SGA neonates could be the result of an increased conversion of cortisol to cortisone (22).

\section{DISCUSSION}

We have shown that the hypothalamic-pituitary-adrenal (HPA) axis system of SGA neonates does not adequately react in response to the application of a stress stimulus. Experiments in fetal sheep showed that the cortisol responses to acute stresses are often blunted during suboptimal intrauterine conditions in late gestation (23). In the presence of adverse intrauterine conditions, decreases in the mRNA content of hypothalamic corticotrophin-releasing hormone, pituitary glucocorticoid receptor, and adrenal ACTH (ACTH) receptor have been found (11), which will impair feedback regulation of the axis. These observations suggest that adverse intrauterine conditions alter both the set point and sensitivity of the fetal HPA axis. Alterations of the HPA axis activity in human adults born SGA have been reported repeatedly. Thus, birth weight has been shown to be inversely correlated with basal cortisol levels in independent populations (9). Furthermore, cortisol responses to pharmacological ACTH challenge $(24,25)$ and after psychosocial stress $(26)$ were exaggerated in adults born with LBW. Others, however, did not find a clear inverse correlation between birth weight and HPA activity (27). These inconsistent results may reflect large heterogeneities in the populations studied, often a mixture of premature infants, infants with uncertain gestational ages, LBW fetuses, fetuses with IUGR as well as different experimental settings.

Although the majority of studies in humans provide strong evidence for an alteration of the HPA axis set point and feedback in adults born SGA, these findings do not necessarily represent etiologic factors involved in the concept of fetal programming especially considering the interdependence of the pituitary-adrenocortical response with other neuroendocrine regulatory systems. Moreover, the influence of environmental factors on alterations of the HPA system remains unknown, because this factor was not controlled in most of the above-mentioned studies. To elucidate a putative intrauterine induction of permanent alteration of the HPA-axis system balance, we focused on HPA-axis activity and development in the early postnatal period. In our study, we were able to demonstrate that the stress response is altered in SGA neonates on the 4th day of life. It is difficult to exactly define an adverse intrauterine environment for the fetus. Fetuses with IUGR are not necessarily SGA and the definition of IUGR varies considerably in the literature. The definition of SGA is unequivocal and is commonly referred to as a weight below the 10th percentile for gestational age. If the definition threshold is set low and fetuses with aneuploidies and infections are excluded, a relatively homogenous population of fetuses experiencing adverse intrauterine conditions is left. In our study, we adopted a threshold for SGA definition as birth weight below the 5th percentile. The median birth weight of the SGA newborns in our study corresponds to the 1.6th percentile defining a relatively homogeneous cohort of growth-restricted neonates. The majority of epidemiologic studies have correlated just LBW with the risk for hypertension (28). One may expect an even more pronounced effect if only infants with proven intrauterine compromise could have been included in these studies.

A strength of our study population is that, in contrast to most studies, maternal influencing factors, such as nicotine and alcohol consumption during pregnancy, and antenatal corticosteroid administration that have been shown to induce growth restriction, but may at the same time have independent effects on the HPA axis of the mother and child (29-34), have all been excluded in our study.

The heel-prick test has been shown to be a significant stress event for the newborn, resulting in HPA axis activation as indicated by significantly increasing cortisol levels in response to the test (35). We found a wide variation in cortisol levels between individual infants. This variation has been observed in other studies as well. Nonetheless, variations over time in the same individual have not been shown to vary significantly $(17,36)$.

Few studies analyzed the activity of the human HPA axis in the adolescent and perinatal period. In SGA children, increased plasma and urinary glucocorticoid levels were found at the age of $9 \mathrm{yr}$ (37), others, however, did not observe a correlation between basal serum cortisol levels and rhythms during a 24-h period in 8-10-y-old children born SGA (38).

To our knowledge, the present study is the first to analyze HPA reactivity in healthy human SGA neonates. Experiments in neonates provide the possibility to study the persistence of intrauterine-induced alteration of the HPA axis into the postnatal period and to differentiate them from changes, which are only transiently altered as a compensatory mechanism during intrauterine undernutrition. At the same time, long-term effects resulting from these primary alterations do not yet have the time to develop. Furthermore, the neonatal period may represent an intermediate stage of HPA axis development still susceptible to plastic changes.

The absent stress response in SGA neonates in our study was rather surprising. A delay in the maturation of the HPA system as a possible explanation is rather unlikely, because premature AGA newborns at $34 \mathrm{wk}$ of gestation already display normal stress reactivity (31). Furthermore, maturation of cerebral structures in SGA fetuses is considered to be rather accelerated (39).

Interestingly, children seem to show reduced cortisol responses to stress factors during the first year of life $(40,41)$, 
which seems to be a stress hyposensitive period. The absent response to stress induction in our SGA population may therefore reflect an accelerated HPA system maturation in comparison to AGA infants accounting for inadequate time for proper establishment of important developmental steps.

Growth-restricted preterm infants ( $<32$ wk of gestation) seem to have a lower steroidogenic capacity than infants with uninhibited fetal growth in response to ACTH stimulation leading to an insufficient response to stress (42). This may be related to enzyme deficiencies in the adrenal cortex (43). However, ACTH stimulation cannot differentiate adrenal and pituitary causes of a diminished response.

Stress hyposensitive periods at different stages of postnatal development have been observed in animals as well (44). Blunted cortisol responses to ACTH and acute stressors have been observed in fetal sheep during chronic unfavorable intrauterine conditions $(23,45)$. These observations are consistent with changes on the molecular level reflected by alterations in various hypothalamic receptor expression levels (11), resulting in reduced HPA sensitivity. Considering these data, intrauterine fetal stress because of undernutrition may lead to a hyporeactivity of the HPA system to stressors in early life, but seems to be modified by postnatal factors. If this notion could be confirmed, HPA system plasticity during early life would provide the chance to therapeutically influence the system. Indeed, influencing the HPA system in the postnatal period has been shown to be possible at least in animals (46).

It was suggested that mode of delivery may influence infant HPA axis response in response to different stress levels for up to 2 months of age (47) even though in a larger population this finding was less clear (48). Saliva samples in our study were obtained during the 4th day of life after stabilization of birth-related fluctuations (49). According to a multiple stepwise regression model, mode of delivery does not seem to have an influence on the HPA axis response at least in our collective. Furthermore, there is evidence from animal models that females might be more sensitive to HPA axis programming than males $(50,51)$. However, we did not find a gender difference in HPA axis reactivity in our study. Associations between LBW and increased HPA axis activation have also not been found to be different in adult men and women (52), thereby confirming our results. It seems that gender-specific cortisol reactivity in general depends on different levels of corticosteroid-binding globulin and sex steroids (53). We therefore cannot exclude gender-specific hormonal influences that may manifest during adolescence and adulthood in SGA infants.

Our data only provide a glimpse of HPA axis alteration in the SGA neonate. We do not know whether these alterations persist or how they change during life. Nevertheless, they represent a prerequisite if intrauterine conditions per se are to play a role in the etiology of adult diseases. Prospective long-term investigations over years are required to prove this hypothesis.

In conclusion, stress response in SGA infants seems to be abnormal beginning as early as the neonatal period. However, the characteristics of these abnormalities are changing constantly during the course of life. Apparently, a hyporeactive HPA axis system in early life is converted to a hyper-reactive system, both being unfavorable. The exact mechanisms responsible for these alterations are still elusive. The apparent plasticity of the system provides hope that therapeutic reprogramming of this system may be a realistic long-term objective.

Acknowledgments. We thank Simone Egli for advice in sample collection, Malgorzata Roos for statistical advice, Seema Das-Kundu for critical reading of the manuscript, and Roland Zimmermann for support.

\section{REFERENCES}

1. Stein CE, Fall CH, Kumaran K, Osmond C, Cox V, Barker DJ 1996 Fetal growth and coronary heart disease in south India. Lancet 348:1269-1273

2. Curhan GC, Willett WC, Rimm EB, Spiegelman D, Ascherio AL, Stampfer MJ 1996 Birth weight and adult hypertension, diabetes mellitus, and obesity in US men. Circulation 94:3246-3250

3. Pine D, Charney D 2002 Children, stress, and sensitization: an integration of basic and clinical research on emotion? Biol Psychiatry 52:773

4. Matthews SG 2002 Early programming of the hypothalamo-pituitary-adrenal axis. Trends Endocrinol Metab 13:373-380

5. Young JB 2002 Programming of sympathoadrenal function. Trends Endocrinol Metab 13:381-385

6. Jones A, Beda A, Ward AM, Osmond C, Phillips DI, Moore VM, Simpson DM 2007 Size at birth and autonomic function during psychological stress. Hypertension 49:548-555

7. Phillips DI, Barker DJ 1997 Association between low birthweight and high resting pulse in adult life: is the sympathetic nervous system involved in programming the insulin resistance syndrome? Diabet Med 14:673-677

8. Phillips DI, Jones A 2006 Fetal programming of autonomic and HPA function: do people who were small babies have enhanced stress responses? J Physiol 572:45-50

9. Phillips DI, Walker BR, Reynolds RM, Flanagan DE, Wood PJ, Osmond C, Barker DJ, Whorwood CB 2000 Low birth weight predicts elevated plasma cortisol concentrations in adults from 3 populations. Hypertension 35:1301-1306

10. Barker DJ, Osmond C, Golding J, Kuh D, Wadsworth ME 1989 Growth in utero, blood pressure in childhood and adult life, and mortality from cardiovascular disease. BMJ 298:564-567

11. Challis JR, Sloboda D, Matthews SG, Holloway A, Alfaidy N, Patel FA, Whittle W, Fraser M, Moss TJ, Newnham J 2001 The fetal placental hypothalamic-pituitaryadrenal (HPA) axis, parturition and post natal health. Mol Cell Endocrinol 185:135144

12. Sapolsky RM, Romero LM, Munck AU 2000 How do glucocorticoids influence stress responses? Integrating permissive, suppressive, stimulatory, and preparative actions. Endocr Rev 21:55-89

13. Miller GE, Cohen S, Ritchey AK 2002 Chronic psychological stress and the regulation of pro-inflammatory cytokines: a glucocorticoid-resistance model. Health Psychol 21:531-541

14. Gould E, Tanapat P 1999 Stress and hippocampal neurogenesis. Biol Psychiatry 46:1472-1479

15. Voigt M, Friese K, Schneider KT, Jorch G, Hesse V 2002 [Kurzmitteilung zu den Perzentilwerten für die Körpermaße Neugeborener]. Geburtsh Frauenheilk 62:274276

16. de Weerth C, Zijl RH, Buitelaar JK 2003 Development of cortisol circadian rhythm in infancy. Early Hum Dev 73:39-52

17. Gunnar MR 1992 Reactivity of the hypothalamic-pituitary-adrenocortical system to stressors in normal infants and children. Pediatrics 90:491-497

18. Calixto C, Martinez FE, Jorge SM, Moreira AC, Martinelli CE Jr 2002 Correlation between plasma and salivary cortisol levels in preterm infants. J Pediatr 140:116118

19. Gunnar MR 1989 Studies of the human infant's adrenocortical response to potentially stressful events. New Dir Child Dev:3-18

20. Rauh M, Groschl M, Rascher W, Dorr HG 2006 Automated, fast and sensitive quantification of 17 alpha-hydroxy-progesterone, androstenedione and testosterone by tandem mass spectrometry with on-line extraction. Steroids 71:450-458

21. Altman D 1991 Practical Statistics for Medical Research. London: Chapman \& Hall

22. Dotsch J, Hohenberger I, Peter M, Sippell W, Dorr HG 2000 Evidence for change of 11 beta-hydroxysteroid dehydrogenase activity during infancy and childhood. Pediatr Res 48:697-700

23. McMillen IC, Adams MB, Ross JT, Coulter CL, Simonetta G, Owens JA, Robinson JS, Edwards LJ 2001 Fetal growth restriction: adaptations and consequences. Reproduction 122:195-204

24. Levitt NS, Lambert EV, Woods D, Hales CN, Andrew R, Seckl JR 2000 Impaired glucose tolerance and elevated blood pressure in low birth weight, nonobese, young south african adults: early programming of cortisol axis. J Clin Endocrinol Metab 85:4611-4618

25. Reynolds RM, Walker BR, Syddall HE, Andrew R, Wood PJ, Whorwood CB, Phillips DI 2001 Altered control of cortisol secretion in adult men with low birth weight and cardiovascular risk factors. J Clin Endocrinol Metab 86:245-250

26. Wust S, Entringer S, Federenko IS, Schlotz W, Hellhammer DH 2005 Birth weight is associated with salivary cortisol responses to psychosocial stress in adult life. Psychoneuroendocrinology 30:591-598 
27. Kajantie E, Eriksson J, Osmond C, Wood PJ, Forsen T, Barker DJ, Phillips DI 2004 Size at birth, the metabolic syndrome and 24-h salivary cortisol profile. Clin Endocrinol (Oxf) 60:201-207

28. Davies AA, Smith GD, May MT, Ben-Shlomo Y 2006 Association between birth weight and blood pressure is robust, amplifies with age, and may be underestimated. Hypertension 48:431-436

29. Rohleder N, Kirschbaum C 2006 The hypothalamic-pituitary-adrenal (HPA) axis in habitual smokers. Int J Psychophysiol 59:236-243

30. Chen M, Wang T, Liao ZX, Pan XL, Feng YH, Wang H 2007 Nicotine-induced prenatal overexposure to maternal glucocorticoid and intrauterine growth retardation in rat. Exp Toxicol Pathol 59:245-251

31. Davis EP, Townsend EL, Gunnar MR, Georgieff MK, Guiang SF, Ciffuentes RF, Lussky RC 2004 Effects of prenatal betamethasone exposure on regulation of stress physiology in healthy premature infants. Psychoneuroendocrinology 29:1028-1036

32. Davis EP, Townsend EL, Gunnar MR, Guiang SF, Lussky RC, Cifuentes RF, Georgieff MK 2006 Antenatal betamethasone treatment has a persisting influence on infant HPA axis regulation. J Perinatol 26:147-153

33. Seckl JR, Holmes MC 2007 Mechanisms of disease: glucocorticoids, their placental metabolism and fetal 'programming' of adult pathophysiology. Nat Clin Pract Endocrinol Metab 3:479-488

34. Ogilvie KM, Rivier C 1997 Prenatal alcohol exposure results in hyperactivity of the hypothalamic-pituitary-adrenal axis of the offspring: modulation by fostering at birth and postnatal handling. Alcohol Clin Exp Res 21:424-429

35. Mantagos S, Koulouris A, Vagenakis A 1991 A simple stress test for the evaluation of hypothalamic-pituitary-adrenal axis during the first 6 months of life. J Clin Endocrinol Metab 72:214-216

36. Peters KL 1998 Neonatal stress reactivity and cortisol. J Perinat Neonatal Nurs 11:45-59

37. Clark PM, Hindmarsh PC, Shiell AW, Law CM, Honour JW, Barker DJ 1996 Size at birth and adrenocortical function in childhood. Clin Endocrinol (Oxf) 45:721-726

38. Dahlgren J, Boguszewski M, Rosberg S, Albertsson-Wikland K 1998 Adrenal steroid hormones in short children born small for gestational age. Clin Endocrinol (Oxf) 49:353-361

39. Amiel-Tison C, Pettigrew AG 1991 Adaptive changes in the developing brain during intrauterine stress. Brain Dev 13:67-76

40. Gunnar MR, Brodersen L, Krueger K, Rigatuso J 1996 Dampening of adrenocortical responses during infancy: normative changes and individual differences. Child Dev 67:877-889
41. Larson MC, White BP, Cochran A, Donzella B, Gunnar M 1998 Dampening of the cortisol response to handling at 3 months in human infants and its relation to sleep, circadian cortisol activity, and behavioral distress. Dev Psychobiol 33:327-337

42. Bolt RJ, van Weissenbruch MM, Popp-Snijders C, Sweep CG, Lafeber HN, Delemarre-van de Waal HA 2002 Fetal growth and the function of the adrenal cortex in preterm infants. J Clin Endocrinol Metab 87:1194-1199

43. Parker CR Jr, Buchina ES, Barefoot TK 1994 Abnormal adrenal steroidogenesis in growth-retarded newborn infants. Pediatr Res 35:633-636

44. Rosenfeld P, Suchecki D, Levine S 1992 Multifactorial regulation of the hypothalamic-pituitary-adrenal axis during development. Neurosci Biobehav Rev 16:553568

45. Gardner DS, Jamall E, Fletcher AJ, Fowden AL, Giussani DA 2004 Adrenocortical responsiveness is blunted in twin relative to singleton ovine fetuses. J Physiol 557:1021-1032

46. Maccari S, Piazza PV, Kabbaj M, Barbazanges A, Simon H, Le Moal M 1995 Adoption reverses the long-term impairment in glucocorticoid feedback induced by prenatal stress. J Neurosci 15:110-116

47. Taylor A, Fisk NM, Glover V 2000 Mode of delivery and subsequent stress response. Lancet 355:120

48. Miller NM, Fisk NM, Modi N, Glover V 2005 Stress responses at birth: determinants of cord arterial cortisol and links with cortisol response in infancy. BJOG 112:921926

49. Herrington CJ, Olomu IN, Geller SM 2004 Salivary cortisol as indicators of pain in preterm infants: a pilot study. Clin Nurs Res 13:53-68

50. Weinstock M, Matlina E, Maor GI, Rosen H, McEwen BS 1992 Prenatal stress selectively alters the reactivity of the hypothalamic-pituitary adrenal system in the female rat. Brain Res 595:195-200

51. McCormick CM, Smythe JW, Sharma S, Meaney MJ 1995 Sex-specific effects of prenatal stress on hypothalamic-pituitary-adrenal responses to stress and brain glucocorticoid receptor density in adult rats. Brain Res Dev Brain Res 84:55-61

52. Reynolds RM, Walker BR, Syddall HE, Andrew R, Wood PJ, Phillips DI 2005 Is there a gender difference in the associations of birthweight and adult hypothalamicpituitary-adrenal axis activity? Eur J Endocrinol 152:249-253

53. Kudielka BM, Buske-Kirschbaum A, Hellhammer DH, Kirschbaum C 2004 HPA axis responses to laboratory psychosocial stress in healthy elderly adults, younger adults, and children: impact of age and gender. Psychoneuroendocrinology 29:83-98 